\title{
ORTHOGONAL POLYNOMIALS IN SEVERAL VARIABLES FOR MEASURES WITH MASS POINTS
}

\author{
ANTONIA M. DELGADO, LIDIA FERNÁNDEZ, TERESA E. PÉREZ, MIGUEL A. PIÑAR*, \\ AND YUAN XU
}

\begin{abstract}
Let $d \nu$ be a measure in $\mathbb{R}^{d}$ obtained from adding a set of mass points to another measure $d \mu$. Orthogonal polynomials in several variables associated with $d \nu$ can be explicitly expressed in terms of orthogonal polynomials associated with $d \mu$, so are the reproducing kernels associated with these polynomials. The explicit formulas that are obtained are further specialized in the case of Jacobi measure on the simplex, with mass points added on the vertices, which are then used to study the asymptotics kernel functions for $d \nu$.
\end{abstract}

\section{INTRODUCTION}

Let $d \mu$ be a measure on $\mathbb{R}^{d}$ with all finite moments and we assume that $d \mu$ is positive definite in the sense that $\int_{\mathbb{R}^{d}} p^{2}(x) d \mu>0$ for every $p \in \Pi^{d}, p \neq 0$, where $\Pi^{d}$ denotes the space of real polynomials in $d$-variables. Let $\langle\cdot, \cdot\rangle_{\mu}$ denote the inner product defined by

$$
\langle p, q\rangle_{\mu}:=\int_{\mathbb{R}^{d}} p(x) q(x) d \mu(x), \quad p, q \in \Pi^{d} .
$$

Then orthogonal polynomials of several variables with respect to $\langle p, q\rangle_{\mu}$ exist. Let $N \geq 1$ be a positive integer and let $\xi_{1}, \xi_{2}, \ldots, \xi_{N}$ be distinct points in $\mathbb{R}^{d}$. Let $\Lambda$ be a positive definite matrix of size $N \times N$. We define a new inner product $\langle\cdot, \cdot\rangle_{\nu}$ by

$$
\langle p, q\rangle_{\nu}:=\langle p, q\rangle_{\mu}+\left(p\left(\xi_{1}\right), p\left(\xi_{2}\right), \ldots, p\left(\xi_{N}\right)\right) \Lambda\left(q\left(\xi_{1}\right), q\left(\xi_{2}\right), \ldots, q\left(\xi_{N}\right)\right)^{\mathrm{tr}},
$$

where the superscript tr indicates the transpose, which can be defined via an integral as in (1.1) against a measure $d \nu$ that is obtained from adding $N$ mass points to $d \mu$. A typical example is when $\Lambda$ is a diagonal matrix with positive entries. The purpose of this paper is to study orthogonal polynomials with respect to the new inner product $\langle\cdot, \cdot\rangle_{\nu}$.

In the case of one-variable, the first study of orthogonal polynomials for measures with mass points was carried out, as far as we know, by Uvarov ( 9 ), who gave a short discussion on the case of adding a finite set of mass points to a measure and showed how to express the orthogonal polynomials with respect to the new measure in terms of those with respect to the old one. The problem was later revitalized by A. M. Krall ([7]), who considered orthogonal polynomials for measures obtained by adding mass points at the end of the interval on which a continuous measure lives.

2000 Mathematics Subject Classification. 42C05; 33C50.

Key words and phrases. Multivariate orthogonal polynomials, Dirac mass.

* Corresponding author. E-mail: mpinar@ugr.es.

Partially supported by Ministerio de Ciencia y Tecnología (MCYT) of Spain and by the European Regional Development Fund (ERDF) through the grant MTM 2008-06689-C02-02, and Junta de Andalucía, Grupo de Investigación FQM 0229. 
The case of Jacobi measure with additional mass points at the end of $[-1,1]$ was studied in [5], where explicit formulas of orthogonal polynomials were constructed. For Jacobi weight with multiple mass points, it is possible to study asymptotic properties of orthogonal polynomials [4. In the case of several variables, however, only the case of $N=1$ has been studied $[3$.

Our main results contain explicit formulas that express orthogonal polynomials and reproducing kernels with respect to $\langle\cdot, \cdot\rangle_{\nu}$ in terms of those with respect to $\langle\cdot, \cdot\rangle_{\mu}$. These results are stated and proved in Section 2. As an example, we consider the case of Jacobi weight function on the simplex in $\mathbb{R}^{d}$, with mass points added at the vertices, for which our formulas can be further specified and expressed in terms of the classical Jacobi polynomials. The result is then used to study the asymptotic expansion of the Christoffel functions with respect to $\langle\cdot, \cdot\rangle_{\nu}$.

\section{Orthogonal POLYNOMials FOR MeAsures With MASS POINTS}

We start with a short subsection on necessary definitions, for which we follow essentially [2], and prove our main results in the second subsection.

2.1. Preliminary. Through this paper, we will use the standard multi-index notation. Let $\mathbb{N}_{0}$ denote the set of nonnegative integers. For a multi-index $\alpha=$ $\left(\alpha_{1}, \ldots, \alpha_{d}\right) \in \mathbb{N}_{0}^{d}$ and $x=\left(x_{1}, \ldots, x_{d}\right) \in \mathbb{R}^{d}$, a monomial in $d$ variables is defined as $x^{\alpha}=x_{1}^{\alpha_{1}} \cdots x_{d}^{\alpha_{d}}$. The integer $|\alpha|=\alpha_{1}+\cdots+\alpha_{d}$ is called the total degree of $x^{\alpha}$. We denote by $\mathcal{P}_{n}^{d}$ the space of homogeneous polynomials of degree $n$ in $d$-variables, $\mathcal{P}_{n}^{d}:=\operatorname{span}\left\{x^{\alpha}:|\alpha|=n\right\}$, and denote by $\Pi_{n}^{d}$ the space of polynomials of total degree at most $n$. The collection of all polynomials in $d$-variables is $\Pi^{d}$. It is well known that

$$
\operatorname{dim} \Pi_{n}^{d}=\left(\begin{array}{c}
n+d \\
n
\end{array}\right) \quad \text { and } \quad \operatorname{dim} \mathcal{P}_{n}^{d}=\left(\begin{array}{c}
n+d-1 \\
n
\end{array}\right):=r_{n}^{d} .
$$

Let $\langle\cdot, \cdot\rangle_{\mu}$ be the inner product defined in (1.1). A polynomial $p \in \Pi_{n}^{d}$ is orthogonal with respect to (1.1) if

$$
\langle p, q\rangle_{\mu}=0, \quad \forall q \in \Pi_{n-1}^{d} .
$$

Our assumption that $d \mu$ is positive definite implies that orthogonal polynomials with respect to $\langle\cdot, \cdot\rangle_{\mu}$ exist. Let us denote by $\mathcal{V}_{n}^{d}$ the space of orthogonal polynomials of total degree $n$. It follows that $\operatorname{dim} \mathcal{V}_{n}^{d}=r_{n}^{d}$. Let $\left\{P_{\alpha}^{n}\right\}_{|\alpha|=n}$ denote a basis of $\mathcal{V}_{n}^{d}$. It is often convenient to use vector notations introduced in [6] and [10]. Let $\left\{\alpha_{1}, \alpha_{2}, \ldots, \alpha_{r_{n}^{d}}\right\}$ be an enumeration of the set $\left\{\alpha \in \mathbb{N}_{0}^{d}:|\alpha|=n\right\}$ according to a fixed monomial order, say the lexicographical order or the reversed lexicographical order. Then the basis $\left\{P_{\alpha}^{n}\right\}_{|\alpha|=n}$ can be written as

$$
\mathbb{P}_{n}=\left\{P_{\alpha_{1}}^{n}, P_{\alpha_{2}}^{n}, \ldots, P_{\alpha_{r_{n}^{d}}}^{n}\right\} .
$$

We will treat $\mathbb{P}_{n}$ both as a set of functions and as a column vector of functions. As column vectors, the orthogonality of $\left\{P_{\alpha_{j}}^{n}\right\}$ can be expressed as

$$
\left\langle\mathbb{P}_{n}, \mathbb{P}_{m}^{\mathrm{tr}}\right\rangle_{\mu}=\int_{\mathbb{R}^{d}} \mathbb{P}_{n}(x) \mathbb{P}_{m}^{\mathrm{tr}}(x) d \mu= \begin{cases}0, & \text { if } n \neq m, \\ H_{n}, & \text { if } n=m,\end{cases}
$$

where the superscript denotes the transpose (so that $\mathbb{P}^{\text {tr }}$ is a row vector) and $H_{n}$ is a matrix of size $r_{n}^{d} \times r_{n}^{d}$, necessarily symmetric, and in fact a positive definite matrix 
by our assumption on $d \mu$. For convenience, we shall call the system $\left\{\mathbb{P}_{n}\right\}_{n=0}^{\infty}=$ $\left\{P_{\alpha}^{n}:|\alpha|=n, n=0,1, \ldots\right\}$ an orthogonal polynomial system (OPS). If $H_{n}$ is the identity matrix, then $\left\{P_{\alpha}^{n}:|\alpha|=n\right\}$ is an orthonormal basis for $\mathcal{V}_{n}^{d}$ and the OPS is called an orthonormal polynomial system.

Likewise, we can write $\mathbf{x}^{n}:=\left\{x^{\alpha}:|\alpha|=n\right\}=\left\{x^{\alpha_{1}}, x^{\alpha_{2}}, \ldots, x^{\alpha_{n}^{d}}\right\}$ and regard it as a column vector. Since each element in $\mathbb{P}_{n}$ is a polynomial of degree $n$, it can be written as a sum of monomials, which, in vector notation, becomes

$$
\mathbb{P}_{n}=\sum_{j=0}^{n} G_{j, n} \mathbf{x}^{j}, \quad \text { where } \quad G_{j, n} \in \mathcal{M}_{r_{n}^{d} \times r_{j}^{d}},
$$

in which $\mathcal{M}_{p \times q}$ denotes the set of real matrices of size $p \times q$. In particular, $G_{n, n}$ is a square matrix and it is necessarily invertible since $\mathbb{P}_{n}$ is a basis of $\mathcal{V}_{n}^{d}$. We call $G_{n, n}$ the leading coefficient of $\mathbb{P}_{n}$.

With respect to $d \mu$, the reproducing kernel of $\mathcal{V}_{n}^{d}$, denoted by $P_{n}(d \mu ; x, y)$, is defined by $\left\langle P_{n}(d \mu ; x, \cdot), p\right\rangle_{\mu}=p(x), p \in \mathcal{V}_{n}^{d}$. In terms of a basis $\mathbb{P}_{n}$ of $\mathcal{V}_{n}^{d}$, it satisifes

$$
P_{n}(d \mu ; x, y)=\mathbb{P}_{n}^{\text {tr }}(x) H_{n}^{-1} \mathbb{P}_{n}(y) \quad \text { with } \quad H_{n}=\left\langle\mathbb{P}_{n}, \mathbb{P}_{n}\right\rangle_{\mu} .
$$

Similarly, the reproducing kernel of $\Pi_{n}^{d}$, denoted by $K_{n}(d \mu ; x, y)$, is defined by $\left\langle K_{n}(d \mu ; x, \cdot), p\right\rangle_{\mu}=p(x), p \in \Pi_{n}^{d}$, and satisfies

$$
K_{n}(d \mu ; x, y)=\sum_{j=0}^{n} P_{j}(d \mu ; x, y), \quad n \geq 0 .
$$

Since the definitions of $P_{n}(d \mu ; x, y)$ and $K_{n}(d \mu ; x, y)$ are independent of the choice of a particular basis, (see [2, Theorem 3.5.1]), it is often more convenient to work with an orthonormal basis. The kernel $K_{n}(d \mu ; x, y)$ plays an important role in studying Fourier orthogonal expansions, as it is the kernel function of the partial sum operator. The reciprocal of $K_{n}(d \mu ; x, x)$ is called Christoffel function, denoted by $\Lambda_{n}(x)$, and it satisfies

$$
\Lambda_{n}(x):=\frac{1}{K_{n}(d \mu ; x, x)}=\inf _{P(x)=1, P \in \Pi_{n}^{d}} \int_{\mathbb{R}^{d}}|P(y)|^{2} d \mu(y) .
$$

2.2. Main results. Our goal is to study orthogonal polynomials with respect to the inner product $\langle\cdot, \cdot\rangle_{\nu}$ defined in (1.2). Let us recall that $\Lambda$ is a given positive definite matrix of order $N$ and $\left\{\xi_{1}, \xi_{2}, \ldots, \xi_{N}\right\}$ is a set of distinct points in $\mathbb{R}^{d}$. Introducing the notation

$$
\mathbf{p}(\xi)=\left\{p\left(\xi_{1}\right), p\left(\xi_{2}\right), \ldots, p\left(\xi_{N}\right)\right\},
$$

and regarding it also as a column vector, we can then rewrite the inner product $\langle\cdot, \cdot\rangle_{\nu}$ in (1.2) as

$$
\langle p, q\rangle_{\nu}=\langle p, q\rangle_{\mu}+\mathbf{p}(\xi)^{\operatorname{tr}} \Lambda \mathbf{q}(\xi),
$$

where $\langle\cdot, \cdot\rangle_{\mu}$ denotes the inner product defined in (1.1). In the case that $\Lambda$ is a diagonal matrix, $\Lambda=\operatorname{diag}\left\{\lambda_{1}, \ldots, \lambda_{N}\right\}$, the inner product $\langle\cdot, \cdot\rangle_{\nu}$ takes the form

$$
\langle p, q\rangle_{\nu}=\langle p, q\rangle_{\mu}+\sum_{j=1}^{N} \lambda_{j} p\left(\xi_{j}\right) q\left(\xi_{j}\right) .
$$

Our first result shows that orthogonal polynomials with respect to $\langle p, q\rangle_{\nu}$ can be derived in terms of those with respect to $\langle p, q\rangle_{\mu}$. The statement and the proof of 
this result relies heavily on the vector-matrix notation. To facilitate the study, we shall introduce several new notations.

Throughout this section, we shall fix $\mathbb{P}_{n}$ as an orthonormal basis for $\mathcal{V}_{n}^{d}$ associated with $d \mu$. We denote by $\mathbf{P}_{n}(\xi)$ the matrix that has $\mathbb{P}_{n}\left(\xi_{i}\right)$ as columns,

$$
\mathrm{P}_{n}(\xi):=\left(\mathbb{P}_{n}\left(\xi_{1}\right)\left|\mathbb{P}_{n}\left(\xi_{2}\right)\right| \ldots \mid \mathbb{P}_{n}\left(\xi_{N}\right)\right) \in \mathcal{M}_{r_{n}^{d} \times N}
$$

denote by $\mathbf{K}_{n-1}$ the matrix whose entries are $K_{n-1}\left(d \mu ; \xi_{i}, \xi_{j}\right)$,

$$
\mathbf{K}_{n-1}:=\left(K_{n-1}\left(d \mu ; \xi_{i}, \xi_{j}\right)\right)_{i, j=1}^{N} \in \mathcal{M}_{N \times N},
$$

and, finally, denote by $\mathbb{K}_{n-1}(\xi, x)$ the vector of functions

$$
\mathbb{K}_{n-1}(\xi, x)=\left\{K_{n-1}\left(d \mu ; \xi_{1}, x\right), K_{n-1}\left(d \mu ; \xi_{2}, x\right), \ldots, K_{n-1}\left(d \mu ; \xi_{N}, x\right)\right\},
$$

which we again regard as a column vector.

From the fact that $K_{n}(d \mu ; x, y)-K_{n-1}(d \mu ; x, y)=P_{n}(d \mu ; x, y)$, we have immediately the following relations,

$$
\begin{aligned}
& \mathbf{P}_{n}^{\operatorname{tr}}(\xi) \mathbb{P}_{n}(x)=\mathbb{K}_{n}(\xi, x)-\mathbb{K}_{n-1}(\xi, x), \\
& \mathbf{P}_{n}^{\operatorname{tr}}(\xi) \mathbf{P}_{n}(\xi)=\mathbf{K}_{n}-\mathbf{K}_{n-1},
\end{aligned}
$$

which will be used below. Let $I_{N}$ denote the identity matrix of order $N$.

Lemma 2.1. The matrix $I_{N}+\Lambda \mathbf{K}_{n-1}$ is invertible.

Proof. First we show that the matrix $\mathbf{K}_{n-1}$ is positive definite, By the definition of $K_{n}(d \mu ; \cdot, \cdot)$, for every $\mathbf{c} \in \mathbb{R}^{N}, \mathbf{c} \neq 0$, we have

$$
\mathbf{c}^{\mathrm{tr}} \mathbf{K}_{n-1} \mathbf{c}=\sum_{|\alpha| \leq n-1} \sum_{i, j=1}^{N} c_{i} c_{j} P_{\alpha}\left(\xi_{i}\right) P_{\alpha}\left(\xi_{j}\right)=\sum_{|\alpha| \leq n-1}\left|\sum_{j=0}^{N} c_{j} P_{\alpha}\left(\xi_{j}\right)\right|^{2}>0,
$$

so that $\mathbf{K}_{n-1}$ is positive definite. The matrix $\Lambda$ is also positive definite, by assumption, so that it is invertible. Since $\Lambda^{-1}\left(I_{N}+\Lambda \mathbf{K}_{n-1}\right)=\Lambda^{-1}+\mathbf{K}_{n-1}$, we see that it is positive definite as well, hence invertible. Consequently, $I_{N}+\Lambda \mathbf{K}_{n-1}$ is invertible.

We are now ready to state and prove our first main result.

Theorem 2.2. Define a polynomial system $\left\{\mathbb{Q}_{n}\right\}_{n \geq 0}$ by $\mathbb{Q}_{0}(x):=\mathbb{P}_{0}(x)$ and

$$
\mathbb{Q}_{n}(x)=\mathbb{P}_{n}(x)-\mathrm{P}_{n}(\xi)\left(I_{N}+\Lambda \mathbf{K}_{n-1}\right)^{-1} \Lambda \mathbb{K}_{n-1}(\xi, x), \quad n \geq 1 .
$$

Then $\left\{\mathbb{Q}_{n}\right\}_{n \geq 0}$ is a sequence of orthogonal polynomials with respect to $\langle\cdot, \cdot\rangle_{\nu}$ defined in (1.2). Conversely, any sequence of orthogonal polynomials with respect to (1.2) can be expressed as in (2.7).

Proof. Let us assume that $\left\{\mathbb{Q}_{n}\right\}_{n \geq 0}$ is an OPS with respect to $\langle\cdot, \cdot\rangle_{\nu}$ and $\mathbb{Q}_{n}$ has the same leading coefficient as $\mathbb{P}_{n}$, which implies, in particular, that $\mathbb{Q}_{0}$ is a constant and $\mathbb{Q}_{0}=\mathbb{P}_{0}$. We show that $\mathbb{Q}_{n}$ satisfies (2.7). By the assumption, the components of $\mathbb{Q}_{n}-\mathbb{P}_{n}$ are elements in $\Pi_{n-1}^{d}$ for $n \geq 1$. Since $\left\{\mathbb{P}_{n}\right\}_{n \geq 0}$ is a basis of $\Pi^{d}$, we can express these components as linear combinations of orthogonal polynomials in $\mathbb{P}_{0}, \mathbb{P}_{1}, \ldots, \mathbb{P}_{n-1}$. In vector-matrix notation, this means that

$$
\mathbb{Q}_{n}(x)=\mathbb{P}_{n}(x)+\sum_{j=0}^{n-1} M_{j}^{n} \mathbb{P}_{j}(x),
$$


where $M_{j}^{n}$ are matrices of size $r_{n}^{d} \times r_{j}^{d}$. These coefficient matrices can be determined from the orthonormality of $\mathbb{P}_{n}$ and $\mathbb{Q}_{n}$. Indeed, by the orthogonality of $\mathbb{Q}_{n}$, $\left\langle\mathbb{Q}_{n}, \mathbb{P}_{j}\right\rangle_{\nu}=0$ for $0 \leq j \leq n-1$, which shows, by the definition of $\langle\cdot, \cdot\rangle_{\nu}$ and the fact that $\mathbb{P}_{j}$ is orthonormal,

$$
M_{j}^{n}=\left\langle\mathbb{Q}_{n}, \mathbb{P}_{j}^{\mathrm{tr}}\right\rangle_{\mu}=-\mathrm{Q}_{n}(\xi)^{\mathrm{tr}} \Lambda \mathrm{P}_{n}(\xi),
$$

where $\mathbf{P}_{n}(\xi)$ is defined as in (2.2) and $\mathbf{Q}_{n}(\xi)=\left\{\mathbb{Q}_{n}\left(\xi_{1}\right)\left|\mathbb{Q}_{n}\left(\xi_{2}\right)\right| \ldots \mid \mathbb{Q}_{n}\left(\xi_{N}\right)\right\}$ in the analogous matrix with $\mathbb{Q}_{n}\left(\xi_{i}\right)$ as its column vectors. Consequently, we obtain

$$
\begin{aligned}
\mathbb{Q}_{n}(x) & =\mathbb{P}_{n}(x)-\sum_{j=0}^{n-1} \mathrm{Q}_{n}(\xi) \Lambda \mathrm{P}_{j}^{\operatorname{tr}}(\xi) \mathbb{P}_{j}(x) \\
& =\mathbb{P}_{n}(x)-\mathrm{Q}_{n}(\xi) \Lambda \mathbb{K}_{n-1}(\xi, x) .
\end{aligned}
$$

where the second equation follows from the relation (2.5), which leads to a telescoping sum that sums up to $\mathbb{K}_{n-1}(\xi, x)$. Setting $x=\xi_{i}$, we obtain

$$
\mathbb{Q}_{n}\left(\xi_{i}\right)=\mathbb{P}_{n}\left(\xi_{i}\right)-\mathrm{Q}_{n}(\xi) \Lambda \mathbb{K}_{n-1}\left(\xi, \xi_{i}\right), \quad 1 \leq i \leq N,
$$

which leads to, by the definition of $\mathbf{K}_{n-1}$ at (2.3), that

$$
\mathbf{Q}_{n}(\xi)=\mathbf{P}_{n}(\xi)-\mathbf{Q}_{n}(\xi) \Lambda \mathbf{K}_{n-1} .
$$

Solving for $\mathrm{Q}_{n}(\xi)$ from the above equation gives

$$
\mathrm{Q}_{n}(\xi)=\mathrm{P}_{n}(\xi)\left(I_{N}+\Lambda \mathbf{K}_{n-1}\right)^{-1} .
$$

Substituting this expression into (2.8) establishes (2.7).

Conversely, if we define polynomials $\mathbb{Q}_{n}$ by (2.7), the above proof shows that $\mathbb{Q}_{n}$ is orthogonal with respect to $\langle\cdot, \cdot\rangle_{\nu}$. Since $\mathbb{Q}_{n}$ and $\mathbb{P}_{n}$ have the same leading coefficient, it is evident that $\left\{\mathbb{Q}_{n}\right\}_{n \geq 0}$ is an OPS in $\Pi^{d}$.

Let $\left\{\mathbb{Q}_{n}\right\}_{n \geq 0}$ be an OPS with respect to (1.2) as in Theorem 2.2. In general, $\mathbb{Q}_{n}$ is not orthonormal. We denote, in the rest of this section,

$$
H_{n}:=\left\langle\mathbb{Q}_{n}, \mathbb{Q}_{n}^{\text {tr }}\right\rangle_{\nu}
$$

Then $H_{n}$ is a positive definite matrix. It turns out that both $H_{n}$ and $H_{n}^{-1}$ can be expressed in terms of matrices that involve only $\left\{\mathbb{P}_{j}\right\}_{j \geq 0}$.

Proposition 2.3. For $n \geq 0$,

$$
\begin{aligned}
H_{n} & =I_{r_{n}^{d}}+\mathrm{P}_{n}(\xi)\left(I_{N}+\Lambda \mathbf{K}_{n-1}\right)^{-1} \Lambda \mathrm{P}_{n}^{\operatorname{tr}}(\xi), \\
H_{n}^{-1} & =I_{r_{n}^{d}}-\mathrm{P}_{n}(\xi)\left(I_{N}+\Lambda \mathbf{K}_{n}\right)^{-1} \Lambda \mathrm{P}_{n}^{\mathrm{tr}}(\xi) .
\end{aligned}
$$

Proof. Since $\mathbb{P}_{n}$ is orthonormal, $\left\langle\mathbb{P}_{n}, \mathbb{P}_{n}^{\mathrm{tr}}\right\rangle_{\mu}=I_{r_{n}^{d}}$. From (2.7) and (2.9) we obtain

$$
\begin{aligned}
H_{n} & =\left\langle\mathbb{Q}_{n}, \mathbb{Q}_{n}^{\mathrm{tr}}\right\rangle_{\nu}=\left\langle\mathbb{Q}_{n}, \mathbb{P}_{n}^{\mathrm{tr}}\right\rangle_{\nu}=\left\langle\mathbb{Q}_{n}, \mathbb{P}_{n}^{\mathrm{tr}}\right\rangle_{\mu}+\mathrm{Q}_{n}(\xi) \Lambda \mathrm{P}_{n}^{\mathrm{tr}}(\xi) \\
& =I_{r_{n}^{d}}+\mathrm{P}_{n}(\xi)\left(I_{N}+\Lambda \mathbf{K}_{n-1}\right)^{-1} \Lambda \mathrm{P}_{n}^{\mathrm{tr}}(\xi),
\end{aligned}
$$

which proves (2.10). In order to establish (2.11), we need to verify that

$$
H_{n}\left(I_{r_{n}^{d}}-\mathrm{P}_{n}(\xi)\left(I_{N}+\Lambda \mathbf{K}_{n}\right)^{-1} \Lambda \mathrm{P}_{n}^{\mathrm{tr}}(\xi)\right)=I_{r_{n}^{d}},
$$

which, by (2.10) and after simplification, reduces to the following equation,

$$
\begin{aligned}
\left(I_{N}+\Lambda \mathbf{K}_{n-1}\right)^{-1} \Lambda \mathrm{P}_{n}(\xi)^{\operatorname{tr}} \mathrm{P}_{n}(\xi)\left(I_{N}+\Lambda \mathbf{K}_{n}\right)^{-1} \\
=\left(I_{N}+\Lambda \mathbf{K}_{n-1}\right)^{-1}-\left(I_{N}+\Lambda \mathbf{K}_{n}\right)^{-1} .
\end{aligned}
$$

Using (2.6), the above equation can be verified by a simple computation. 
Our next result gives explicit formulas for the reproducing kernels associated with $\langle\cdot, \cdot\rangle_{\nu}$, which we denote by

$$
P_{j}(d \nu ; x, y):=\mathbb{Q}_{j}^{\mathrm{tr}}(x) H_{j}^{-1} \mathbb{Q}_{j}(y) \quad \text { and } \quad K_{n}(d \nu ; x, y):=\sum_{j=0}^{n} P_{j}(d \nu ; x, y) .
$$

Theorem 2.4. For $j \geq 0$,

$$
\begin{aligned}
P_{j}(d \nu ; x, y)=P_{j}(d \mu ; x, y) & -\mathbb{K}_{j}^{\mathrm{tr}}(\xi, x)\left(I_{N}+\Lambda \mathbf{K}_{j}\right)^{-1} \Lambda \mathbb{K}_{j}(\xi, y) \\
& +\mathbb{K}_{j-1}^{\mathrm{tr}}(\xi, x)\left(I_{N}+\Lambda \mathbf{K}_{j-1}\right)^{-1} \Lambda \mathbb{K}_{j-1}(\xi, y) .
\end{aligned}
$$

Furthermore, for $n \geq 0$,

$$
K_{n}(d \nu ; x, y)=K_{n}(d \mu ; x, y)-\mathbb{K}_{n}^{\mathrm{tr}}(\xi, x)\left(I_{N}+\Lambda \mathbf{K}_{n}\right)^{-1} \Lambda \mathbb{K}_{n}(\xi, y) .
$$

Proof. Since $\Lambda^{-1}\left(I_{N}+\Lambda \mathbf{K}_{j-1}\right)=\Lambda^{-1}+\mathbf{K}_{j-1}$ is a symmetric matrix, so is $\left(I_{N}+\right.$ $\left.\Lambda \mathbf{K}_{j-1}\right)^{-1} \Lambda$. Using this fact, it follows from (2.7) and (2.11) that

$$
\begin{aligned}
\mathbb{Q}_{j}^{\operatorname{tr}}(x) H_{j}^{-1}= & \mathbb{P}_{j}^{\operatorname{tr}}(x)-\mathbb{P}_{j}^{\operatorname{tr}}(x) \mathrm{P}_{j}(\xi)\left(I-\Lambda \mathbf{K}_{j}\right)^{-1} \Lambda \mathrm{P}_{j}^{\operatorname{tr}}(\xi) \\
& -\mathbb{K}_{j-1}^{\operatorname{tr}}(\xi, x)\left(I_{N}+\Lambda \mathbf{K}_{j-1}\right)^{-1} \Lambda \mathrm{P}_{j}^{\operatorname{tr}}(\xi) \\
& -\mathbb{K}_{j-1}^{\operatorname{tr}}(\xi, x)\left(I_{N}+\Lambda \mathbf{K}_{j-1}\right)^{-1} \Lambda \mathrm{P}_{j}^{\operatorname{tr}}(\xi) \mathrm{P}_{j}(\xi)\left(I+\Lambda \mathbf{K}_{j}\right)^{-1} \mathrm{P}_{j}^{\operatorname{tr}}(\xi),
\end{aligned}
$$

which simplifies to, upon using (2.12) and (2.5),

$$
\begin{aligned}
\mathbb{Q}_{j}^{\mathrm{tr}}(x) H_{j}^{-1}= & \mathbb{P}_{j}^{\mathrm{tr}}(x)-\mathbb{P}_{j}^{\mathrm{tr}}(x) \mathrm{P}_{j}(\xi)\left(I-\Lambda \mathbf{K}_{j}\right)^{-1} \Lambda \mathrm{P}_{j}^{\mathrm{tr}}(\xi) \\
& -\mathbb{K}_{j-1}^{\mathrm{tr}}(\xi, x)\left(I_{N}+\Lambda \mathbf{K}_{j-1}\right)^{-1} \Lambda \mathrm{P}_{j}^{\mathrm{tr}}(\xi) \\
= & \mathbb{P}_{j}^{\operatorname{tr}}(x)-\mathbb{K}_{j}^{\mathrm{tr}}(\xi, x)\left(I_{N}+\Lambda \mathbf{K}_{j}\right)^{-1} \Lambda \mathrm{P}_{j}^{\mathrm{tr}}(\xi) .
\end{aligned}
$$

Using again (2.7) and (2.5), we then obtain

$$
\begin{aligned}
\mathbb{Q}_{j}^{\operatorname{tr}}(x) & H_{j}^{-1} \mathbb{Q}_{j}(y)=\mathbb{P}_{j}^{\operatorname{tr}}(x) \mathbb{P}_{j}(y) \\
& -\left[\mathbb{K}_{j}^{\operatorname{tr}}(\xi, x)-\mathbb{K}_{j-1}^{\operatorname{tr}}(\xi, x)\right]\left(I_{N}+\Lambda \mathbf{K}_{j-1}\right)^{-1} \Lambda \mathbb{K}_{j-1}(\xi, y) \\
& -\mathbb{K}_{j}^{\operatorname{tr}}(\xi, x)\left(I_{N}+\Lambda \mathbf{K}_{j}\right)^{-1} \Lambda\left[\mathbb{K}_{j}(\xi, y)-\mathbb{K}_{j-1}(\xi, y)\right] \\
& +\mathbb{K}_{j}^{\operatorname{tr}}(\xi, x)\left(I_{N}+\Lambda \mathbf{K}_{j}\right)^{-1} \Lambda \mathrm{P}_{j}^{\operatorname{tr}}(\xi) \mathrm{P}_{j}(\xi)\left(I_{N}+\Lambda \mathbf{K}_{j-1}\right)^{-1} \Lambda \mathbb{K}_{j-1}(\xi, y),
\end{aligned}
$$

which simplifies to (2.13) upon using the identity (2.12).

Finally, summing over (2.13) for $j=0,1, \ldots, n$, we obtain (2.14).

The result in this section can be extended without much difficulty to mass points with derivative values. To be more precise, let $\partial^{\alpha}=\partial_{1}^{\alpha_{1}} \cdots \partial_{d}^{\alpha_{d}}$, where $\partial_{i}=\frac{\partial}{\partial x_{i}}$, and for $\alpha_{i} \in \mathbb{N}_{0}^{d}, i=1,2, \ldots, N$, define

$$
D_{\alpha} \mathbf{p}(\xi):=\left\{\partial^{\alpha_{1}} p\left(\xi_{1}\right), \partial^{\alpha_{2}} p\left(\xi_{2}\right), \ldots, \partial^{\alpha_{N}} p\left(\xi_{N}\right)\right\},
$$

and regard it also as a column vector. Instead of requiring $\xi_{i} \neq \xi_{j}$, we only assume that $\xi_{i} \neq \xi_{j}$ when $\alpha_{i}=\alpha_{j}$. In other word, $\xi_{i}$ and $\xi_{j}$ can be the same as long as $\alpha_{i} \neq \alpha_{j}$. We then consider the inner product defined by

$$
\langle p, q\rangle_{\nu}=\langle p, q\rangle_{\mu}+D_{\alpha} \mathbf{p}(\xi)^{\operatorname{tr}} \Lambda D_{\alpha} \mathbf{q}(\xi) .
$$

When $\alpha_{i}=0$ for all $i$, this is the inner product in (1.2). Other interesting cases include, for example,

$$
\langle p, q\rangle_{\nu}=\langle p, q\rangle_{\mu}+\sum_{j=0}^{N} \lambda_{j} p\left(\xi_{j}\right) q\left(\xi_{j}\right)+\sum_{j=0}^{N} \lambda_{j}^{\prime} \nabla p\left(\xi_{j}\right) \cdot \nabla q\left(\xi_{j}\right) .
$$


Our results in Theorem 2.2. Proposition 2.3 and Theorem 2.4 still hold in this setting, but we need to replace $\mathbf{P}_{n}$ in (2.2), $\mathbf{K}_{n-1}$ in (2.3), and $\mathbb{K}_{n-1}(\xi, x)$ in (2.4) by

$$
\begin{aligned}
\mathrm{P}_{n}^{*}(\xi) & :=\left(\partial^{\alpha_{1}} \mathbb{P}_{n}\left(\xi_{1}\right)\left|\partial^{\alpha_{2}} \mathbb{P}_{n}\left(\xi_{2}\right)\right| \ldots \mid \partial^{\alpha_{d}} \mathbb{P}_{n}\left(\xi_{N}\right)\right) \in \mathcal{M}_{r_{n}^{d} \times N}, \\
\mathbf{K}_{n-1}^{*} & :=\left(\partial_{\{1\}}^{\alpha_{i}} \partial_{\{2\}}^{\alpha_{j}} K_{n-1}^{*}\left(d \mu ; \xi_{i}, \xi_{j}\right)\right)_{i, j=1}^{N} \in \mathcal{M}_{N \times N}, \\
\mathbb{K}_{n-1}^{*}(\xi, x) & =\left\{\partial_{\{1\}}^{\alpha_{1}} K_{n-1}\left(d \mu ; \xi_{1}, x\right), \partial_{\{1\}}^{\alpha_{2}} K_{n-1}\left(d \mu ; \xi_{2}, x\right), \ldots, \partial_{\{1\}}^{\alpha_{d}} K_{n-1}\left(d \mu ; \xi_{N}, x\right)\right\}
\end{aligned}
$$

respectively, where $\partial_{\{1\}}^{\alpha} K_{n}(u, v)$ means that the derivative is taken with respect to $u$ variable.

Theorem 2.5. The results in Theorem 2.2 and Theorem 2.4 hold for the inner product defined in (2.15) when $\mathbf{P}_{\mathbf{n}}, \mathbf{K}_{n-1}$ and $\mathbb{K}_{n-1}(\xi, x)$ are replaced by $\mathbf{P}_{n}^{*}, \mathbf{K}_{n-1}^{*}$ and $\mathbb{K}_{n-1}^{*}(\xi, x)$, respectively.

The proof follows as before almost verbatim with little additional difficulty.

\section{Orthogonal Polynomials on the SimpleX}

In this section we apply the general result in the previous section to orthogonal polynomials on the simplex

$$
T^{d}:=\left\{x=\left(x_{1}, \ldots, x_{d}\right) \in \mathbb{R}^{d}: x_{i} \geq 0,1-|x|_{1} \geq 0\right\}
$$

in $\mathbb{R}^{d}$, where $|x|_{1}=x_{1}+\ldots+x_{d}$.

3.1. Jacobi polynomials on the simplex. We consider the Jacobi weight function

$$
W_{\kappa}(x)=x_{1}^{\kappa_{1}-1 / 2} \cdots x_{d}^{\kappa_{d}-1 / 2}\left(1-|x|_{1}\right)^{\kappa_{d+1}-1 / 2}, \quad \kappa_{i} \geq 0,
$$

on the simplex, where

$$
w_{\kappa}=\frac{\Gamma\left(|\kappa|+\frac{d+1}{2}\right)}{\Gamma\left(\kappa_{1}+\frac{1}{2}\right) \cdots \Gamma\left(\kappa_{d+1}+\frac{1}{2}\right)}, \quad|\kappa|:=\kappa_{1}+\kappa_{2}+\cdots+\kappa_{d+1},
$$

is the normalization constant of $W_{\kappa}$ such that $w_{\kappa} \int_{T^{d}} W_{\kappa}(x) d x=1$. Associated with $W_{\kappa}$, we consider the inner product on the simplex

$$
\langle f, g\rangle=w_{\kappa} \int_{T^{d}} f(x) g(x) W_{\kappa}(x) d x,
$$

which plays the role of $\langle\cdot, \cdot\rangle_{\mu}$ when we deal with the settings of the previous section. For $d=1, W_{\kappa}$ is the classical Jacobi weight function, which has orthogonal polynomials $P_{n}^{\left(\kappa_{1}, \kappa_{2}\right)}(2 t-1)$, where $P_{n}^{(a, b)}$ is the classical Jacobi polynomial of degree $n$ that is orthogonal with respect to $(1-t)^{a}(1+t)^{b}$ on $[-1,1]$ and normalized by $P_{n}^{(a, b)}(1)=\left(\begin{array}{c}n+a \\ n\end{array}\right)$. We shall also denote the orthonormal Jacobi polynomials by $p_{n}^{(a, b)}(t)$. Evidently, $p_{n}^{(a, b)}(t)=c_{n} P_{n}^{(a, b)}(t)$, where the constant $c_{n}$ is given by [8, (4.3.3)].

To state an orthonormal basis for $\mathcal{V}_{n}^{d}$ on the simplex, we follow [2, p. 47] and introduce the following notation. Associated with $x=\left(x_{1}, \ldots, x_{d}\right) \in \mathbb{R}^{d}$, we define by $\mathbf{x}_{j}$ the truncation of $x$, namely

$$
\mathbf{x}_{0}=0, \quad \mathbf{x}_{j}=\left(x_{1}, \ldots, x_{j}\right), \quad 1 \leq j \leq d,
$$


and associated with $\alpha=\left(\alpha_{1}, \ldots, \alpha_{d}\right) \in \mathbb{N}_{0}^{d}$ and $\kappa=\left(\kappa_{1}, \ldots, \kappa_{d+1}\right) \in \mathbb{R}^{d+1}$, we introduce, respectively,

$$
\alpha^{j}:=\left(\alpha_{j}, \ldots, \alpha_{d}\right), \quad 1 \leq j \leq d, \quad \kappa^{j}:=\left(\kappa_{j}, \ldots, \kappa_{d+1}\right), \quad 1 \leq j \leq d+1 .
$$

Then, an orthonormal basis associated with (3.1) is given explicitly by

$$
P_{\alpha}\left(W_{\kappa} ; x\right)=h_{\alpha}^{-1} \prod_{j=1}^{d}\left(\frac{1-\left|\mathbf{x}_{j}\right|_{1}}{1-\left|\mathbf{x}_{j-1}\right|_{1}}\right)^{\left|\alpha^{j+1}\right|} p_{\alpha_{j}}^{\left(a_{j}, b_{j}\right)}\left(\frac{2 x_{j}}{1-\left|\mathbf{x}_{j-1}\right|_{1}}-1\right),
$$

where the parameters $a_{j}$ and $b_{j}$ are given by

$$
a_{j}=2\left|\alpha^{j+1}\right|+\left|\kappa^{j+1}\right|+\frac{d-j-1}{2}, \quad b_{j}=\kappa_{j}-\frac{1}{2},
$$

and $h_{\alpha}$ is the normalizing constant given by

$$
h_{\alpha}^{2}=\frac{\left(|\kappa|+\frac{d+1}{2}\right)_{2|\alpha|}}{\prod_{j=1}^{d}\left(2\left|\alpha^{j+1}\right|+\left|\kappa^{j}\right|+\frac{d-j+2}{2}\right)_{2 \alpha_{j}}},
$$

in which $(a)_{k}:=a(a+1) \ldots(a+k-1)$ denotes the shifted factorial.

In this case, we also have a compact formula for the reproducing kernels, given in terms of the Gegenbauer polynomials $C_{n}^{\lambda}$, which are orthogonal with respect to the weight function $\left(1-t^{2}\right)^{\lambda-1 / 2}$ and normalized by $C_{n}^{\lambda}(1)=\left(\begin{array}{c}n+2 \lambda-1 \\ n\end{array}\right)$. The formula, first derived in [11, Theorem 2.3], is given by

$$
\begin{gathered}
K_{n}\left(W_{\kappa} ; x, y\right)=\frac{1}{2^{d+1}} \int_{[-1,1]^{d+1}} C_{2 n}^{\lambda}\left(\sqrt{x_{1} y_{1}} t_{1}+\cdots+\sqrt{x_{d+1} y_{d+1}} t_{d+1}\right) \\
\quad \times \prod_{j=1}^{d+1} c_{\kappa_{j}}\left(1-t_{j}^{2}\right)^{\kappa_{j}-1} d t
\end{gathered}
$$

where $x_{d+1}=1-|x|_{1}, y_{d+1}=1-|y|_{1}, \lambda:=|\kappa|+\frac{d+1}{2}$, and $c_{\kappa_{j}}=\int_{-1}^{1}\left(1-t_{j}^{2}\right)^{\kappa_{j}-1} d t_{i}$.

Let us denote the standard Euclidean basis of $\mathbb{R}^{d}$ by $\left\{e_{1}, \ldots, e_{d}\right\}$, where $e_{i}=$ $(0, \ldots, 0,1,0 \ldots, 0)$ with the single 1 in the $i$-th position. Furthermore, we set $e_{d+1}=(0, \ldots, 0) \in \mathbb{R}^{d}$. Then $\left\{e_{1}, e_{2}, \ldots, e_{d+1}\right\}$ is the set of vertices of $T^{d}$.

Proposition 3.1. Let $\lambda=|\kappa|+\frac{d+1}{2}$. For $1 \leq i \leq d+1$, we have

$$
K_{n}\left(W_{\kappa} ; x, e_{i}\right)=\frac{1}{2^{d+1}} \frac{(\lambda)_{n}}{\left(\kappa_{i}+1 / 2\right)_{n}} P_{n}^{\left(\lambda-\kappa_{i}-1 / 2, \kappa_{i}-1 / 2\right)}\left(2 x_{i}-1\right) .
$$

In particular, we have

$$
\begin{aligned}
& K_{n}\left(W_{\kappa} ; e_{i}, e_{i}\right)=\frac{1}{2^{d+1}} \frac{(\lambda)_{n}}{n !} \frac{\left(\lambda-\kappa_{i}+1 / 2\right)_{n}}{\left(\kappa_{i}+1 / 2\right)_{n}}, \quad 1 \leq i \leq d+1 . \\
& K_{n}\left(W_{\kappa} ; e_{i}, e_{j}\right)=\frac{(-1)^{n}}{2^{d+1}} \frac{(\lambda)_{n}}{n !}, \quad 1 \leq i, j \leq d+1,
\end{aligned}
$$


Proof. Since $C_{2 n}^{\lambda}$ is an even function, for $1 \leq i \leq d+1$ we deduce from (3.3) that

$$
\begin{aligned}
K_{n}\left(W_{\kappa} ; x, e_{i}\right) & =\frac{c_{\kappa_{i}}}{2^{d+1}} \int_{-1}^{1} C_{2 n}^{\lambda}\left(\sqrt{x_{i}} t_{i}\right)\left(1-t_{i}^{2}\right)^{\kappa_{i}-1} d t_{i} \\
& =\frac{c_{\kappa_{i}}}{2^{d+1}} \int_{-1}^{1} C_{2 n}^{\lambda}\left(\sqrt{x_{i}} t_{i}\right)\left(1-t_{i}\right)^{\kappa_{i}-1}\left(1+t_{i}\right)^{\kappa_{i}} d t_{i} \\
& =\frac{1}{2^{d+1}} V^{\left(\kappa_{i}\right)} C_{2 n}^{\lambda}\left(\sqrt{x_{i}}\right) \\
& =\frac{1}{2^{d+1}} \frac{(\lambda)_{n}}{\left(\kappa_{i}+1 / 2\right)_{n}} P_{n}^{\left(\lambda-\kappa_{i}-1 / 2, \kappa_{i}-1 / 2\right)}\left(2 x_{i}-1\right),
\end{aligned}
$$

where $V^{\left(\kappa_{i}\right)}$ is the operator defined in [2, Definition 1.5.1, p. 24] and the last equality comes from [2, Proposition 1.5.6, p. 27]. In particular, setting $x=e_{j}$ in (3.4) shows that

$$
K_{n}\left(W_{\kappa} ; e_{i}, e_{i}\right)=\frac{1}{2^{d+1}} \frac{(\lambda)_{n}}{\left(\kappa_{i}+1 / 2\right)_{n}} P_{n}^{\left(\lambda-\kappa_{i}-1 / 2, \kappa_{i}-1 / 2\right)}(1)
$$

and, for $i \neq j$,

$$
K_{n}\left(W_{\kappa} ; e_{j}, e_{i}\right)=\frac{1}{2^{d+1}} \frac{(\lambda)_{n}}{\left(\kappa_{i}+1 / 2\right)_{n}} P_{n}^{\left(\lambda-\kappa_{i}-1 / 2, \kappa_{i}-1 / 2\right)}(-1)
$$

from which (3.5) and (3.6) follow from [8, (4.1.1) and (4.1.4)]).

3.2. Orthogonal polynomials on the simplex with mass points. We consider orthogonal polynomials on the simplex for the Jacobi measure with additional mass at each of the vertices of the simplex. In order to preserve symmetry, we shall limit ourself to the situation that every vertex has the same weight $M>0$. In other words, we consider the inner product

$$
\langle f, g\rangle_{\nu}=w_{\kappa} \int_{T^{d}} f(x) g(x) W_{\kappa}(x) d x+M \sum_{i=1}^{d+1} f\left(e_{i}\right) g\left(e_{i}\right), \quad M>0 .
$$

In the language of the inner product (1.2), we assume that $\Lambda$ is a diagonal matrix $\Lambda=M I_{d+1}$ and the inner product take the form of (2.1).

We will further limit ourself to the case that $\kappa_{1}=\kappa_{2}=\cdots=\kappa_{d+1}=\varsigma \geq 0$. Under this assumption,

$$
\lambda=(d+1)(\varsigma+1 / 2)
$$

We further denote

$$
\begin{aligned}
& A_{n}:=K_{n}\left(W_{\kappa} ; e_{i}, e_{i}\right)=\frac{1}{2^{d+1}} \frac{(\lambda)_{n}}{n !} \frac{(\lambda-\varsigma+1 / 2)_{n}}{(\varsigma+1 / 2)_{n}}, \\
& B_{n}:=K_{n}\left(W_{\kappa} ; e_{j}, e_{i}\right)=\frac{(-1)^{n}}{2^{d+1}} \frac{(\lambda)_{n}}{n !}, \quad j \neq i .
\end{aligned}
$$


As a result, we see that the matrix $\mathbf{K}_{n}$ defined in (2.3) is given by

$$
\begin{aligned}
\mathbf{K}_{n} & =\left(K_{n}\left(W_{\kappa} ; e_{i}, e_{j}\right)\right)_{i, j=1}^{d+1}=\left(\begin{array}{cccc}
A_{n} & B_{n} & \cdots & B_{n} \\
B_{n} & A_{n} & \cdots & B_{n} \\
\vdots & \vdots & \ddots & \vdots \\
B_{n} & B_{n} & \cdots & A_{n}
\end{array}\right) \\
& =\left(A_{n}-B_{n}\right) I_{d+1}+B_{n}\left(\begin{array}{ccc}
1 & \cdots & 1 \\
\vdots & & \vdots \\
1 & \cdots & 1
\end{array}\right) \\
& =\left(A_{n}-B_{n}\right) I_{d+1}+B_{n}\left(\begin{array}{c}
1 \\
\vdots \\
1
\end{array}\right)(1, \ldots, 1) .
\end{aligned}
$$

This shows that $\mathbf{K}_{n}$ is a rank one perturbation of the identity matrix and, consequently, the inverse of the matrix $I_{d+1}+\Lambda \mathbf{K}_{n}$ can be easily verified to be

$$
\begin{aligned}
\left(I_{d+1}+\Lambda \mathbf{K}_{n}\right)^{-1} \Lambda= & \frac{M}{\left[1+M\left(A_{n}-B_{n}\right)\right]\left[1+M A_{n}+d M B_{n}\right]} \\
& \times\left[\left(1+M A_{n}+d M B_{n}\right) I_{d+1}-M B_{n}\left(\begin{array}{ccc}
1 & \cdots & 1 \\
\vdots & & \vdots \\
1 & \cdots & 1
\end{array}\right)\right] .
\end{aligned}
$$

As a result, we can now use Theorem 2.2 to derive an explicit orthogonal basis for the inner product (3.7), which is given by

$$
\begin{aligned}
\mathbb{Q}_{n}(x)= & \mathbb{P}_{n}(x)+\frac{M}{1+M\left(A_{n-1}-B_{n-1}\right)} \sum_{i=1}^{d+1} \mathbb{P}_{n}\left(e_{i}\right) K_{n-1}\left(W_{\kappa} ; x, e_{i}\right) \\
- & \frac{M^{2} B_{n-1}}{\left[1+M\left(A_{n-1}-B_{n-1}\right)\right]\left[1+M A_{n-1}+d M B_{n-1}\right]} \times \\
& \times \sum_{i=1}^{d+1} \mathbb{P}_{n}\left(e_{i}\right) \sum_{i=1}^{d+1} K_{n-1}\left(W_{\kappa} ; x, e_{i}\right),
\end{aligned}
$$

where $\left\{\mathbb{P}_{n}\right\}_{n \geq 0}$ denotes the orthonormal polynomial system on the simplex $T^{d}$ given by (3.2). Furthermore, by Theorem 2.4, the reproducing kernel $K_{n}(d \nu ; x, y)$ for $\Pi_{n}^{d}$ under the inner product (3.7) is given by

$$
\begin{aligned}
K_{n}(d \nu ; x, y)= & K_{n}\left(W_{\kappa} ; x, y\right)+\frac{M}{1+M\left(A_{n}-B_{n}\right)} \sum_{i=1}^{d+1} K_{n}\left(W_{\kappa} ; x, e_{i}\right) K_{n}\left(W_{\kappa} ; y, e_{i}\right) \\
- & \frac{M^{2} B_{n}}{\left[1+M\left(A_{n}-B_{n}\right)\right]\left[1+M A_{n}+d M B_{n}\right]} \times \\
& \times \sum_{i=1}^{d+1} K_{n}\left(W_{\kappa} ; x, e_{i}\right) \sum_{i=1}^{d+1} K_{n}\left(W_{\kappa} ; y, e_{i}\right) .
\end{aligned}
$$

The explicit formula of the kernel allows us to derive a sharp estimate for the kernel $K_{n}(d \nu ; x, y)$ from those for $K_{n}\left(W_{\kappa} ; x, y\right)$ and for the Jacobi polynomials. In the case of one variable $(d=1)$, such an estimate has been carried out in 4 . We shall give one result on the strong asymptotic of the Christoffel function with 
respect to $d \nu$ on the simplex $T^{d}$. For this purpose, we will need the following estimate of the Jacobi polynomials ([8, (7.32.5) and (4.1.3)]):

Lemma 3.2. For an arbitrary real number $\alpha$ and $t \in[0,1]$,

$$
\left|P_{n}^{(\alpha, \beta)}(t)\right| \leq c n^{-1 / 2}\left(1-t+n^{-2}\right)^{-(\alpha+1 / 2) / 2} .
$$

The estimate on $[-1,0]$ follows from the fact that $P_{n}^{(\alpha, \beta)}(t)=(-1)^{n} P_{n}^{(\beta, \alpha)}(-t)$.

Note that (3.9) shows that $\left|P_{n}^{(\alpha, \beta)}\right| \leq c n^{-1 / 2}$ uniformly inside a compact subset of $(-1,1)$. We derive the asymptotic for the difference $K_{n}(d \nu ; x, x)-K_{n}\left(W_{\kappa} ; x, x\right)$.

Theorem 3.3. For $x$ in $T^{d}$,

$$
\begin{aligned}
K_{n}(d \nu ; x, x)- & K_{n}\left(W_{\kappa} ; x, x\right)=\frac{1}{2^{d+1}} \frac{\Gamma(\lambda-\varsigma+1 / 2) \Gamma(\varsigma+1 / 2)}{\Gamma(\lambda)} \\
& \times \sum_{i=1}^{d+1}\left[P_{n}^{(\lambda-\varsigma-1 / 2, \varsigma-1 / 2)}\left(2 x_{i}-1\right)\right]^{2}\left(1+\mathcal{O}\left(n^{-1}\right)\right) .
\end{aligned}
$$

In particular, for $x$ in the interior of $T^{d}$,

$$
\lim _{n \rightarrow \infty}\left[K_{n}(d \nu ; x, x)-K_{n}\left(W_{\kappa} ; x, x\right)\right]=0,
$$

and the convergence is uniform in any compact set in the interior of $T^{d}$.

Proof. From (3.8) we deduce

$$
\begin{aligned}
K_{n}(d \nu ; x, x)- & K_{n}\left(W_{\kappa} ; x, x\right)=\frac{M}{1+M\left(A_{n}-B_{n}\right)} \sum_{i=1}^{d+1} K_{n}\left(W_{\kappa} ; x, e_{i}\right)^{2} \\
& -\frac{M^{2} B_{n}}{\left[1+M\left(A_{n}-B_{n}\right)\right]\left[1+M A_{n}+d M B_{n}\right]} \times\left(\sum_{i=1}^{d+1} K_{n}\left(W_{\kappa} ; x, e_{i}\right)\right)^{2} \\
= & \frac{M C_{n}^{2}}{1+M\left(A_{n}-B_{n}\right)} \sum_{i=1}^{d+1}\left[P_{n}^{(\lambda-\varsigma-1 / 2, \varsigma-1 / 2)}\left(2 x_{i}-1\right)\right]^{2} \\
& -\frac{M^{2} C_{n}^{2} B_{n}}{\left[1+M\left(A_{n}-B_{n}\right)\right]\left[1+M A_{n}+d M B_{n}\right]} \times \\
& \times\left(\sum_{i=1}^{d+1} P_{n}^{(\lambda-\varsigma-1 / 2, \varsigma-1 / 2)}\left(2 x_{i}-1\right)\right)^{2}
\end{aligned}
$$

where

$$
C_{n}=\frac{1}{2^{d+1}} \frac{(\lambda)_{n}}{(\varsigma+1 / 2)_{n}} .
$$

By the Stirling formula for the Gamma function (see [1, (6.1.39), p. 257]), we have

$$
\frac{\Gamma(n+a)}{\Gamma(n+1)}=n^{a-1}\left(1+\mathcal{O}\left(n^{-1}\right)\right)
$$

as $n \rightarrow \infty$. Consequently, it is easy to see that the following limit relations hold:

$$
\begin{aligned}
\frac{M C_{n}^{2}}{1+M\left(A_{n}-B_{n}\right)} & =\frac{1}{2^{d+1}} \frac{\Gamma(\lambda-\varsigma+1 / 2) \Gamma(\varsigma+1 / 2)}{\Gamma(\lambda)}\left(1+\mathcal{O}\left(n^{-1}\right)\right), \\
\frac{M B_{n}}{1+M A_{n}+d M B_{n}} & =(-1)^{n} \frac{\Gamma(\lambda-\varsigma+1 / 2)}{\Gamma(\varsigma+1 / 2)} n^{-(\lambda-2 \varsigma-1)}\left(1+\mathcal{O}\left(n^{-1}\right)\right) .
\end{aligned}
$$


Since $\lambda-2 \varsigma-1=(d-1)(\varsigma+1 / 2)>0$ for $d \geq 2$ and

$$
\left(\sum_{i=1}^{d+1} P_{n}^{(\lambda-\varsigma-1 / 2, \varsigma-1 / 2)}\left(2 x_{i}-1\right)\right)^{2} \leq(d+1) \sum_{i=1}^{d+1}\left[P_{n}^{(\lambda-\varsigma-1 / 2, \varsigma-1 / 2)}\left(2 x_{i}-1\right)\right]^{2}
$$

by the Cauchy-Schwarz inequality, it follows readily that

$K_{n}(d \nu ; x, x)-K_{n}\left(W_{\kappa} ; x, x\right)=c_{\lambda, \varsigma} \sum_{i=1}^{d+1}\left[P_{n}^{(\lambda-\varsigma-1 / 2, \varsigma-1 / 2)}\left(2 x_{i}-1\right)\right]^{2}\left(1+\mathcal{O}\left(n^{-1}\right)\right)$,

where $c_{\lambda, \varsigma}$ is the constant

$$
c_{\lambda, \varsigma}=\frac{1}{2^{d+1}} \frac{\Gamma(\lambda-\varsigma+1 / 2) \Gamma(\varsigma+1 / 2)}{\Gamma(\lambda)} .
$$

This is (3.10). If $x$ is in the interior of $T^{d}$, then $\left|P_{n}^{(\lambda-\varsigma-1 / 2, \varsigma-1 / 2)}\left(2 x_{i}-1\right)\right| \leq c n^{-1 / 2}$, so that $K_{n}(d \nu ; x, x)-K_{n}\left(W_{\kappa} ; x, x\right)$ goes to zero as $n \rightarrow \infty$.

The asymptotic of the Christoffel function for $W_{\kappa}$ was studied in 12, where most of the results were for convergence in the interior of $T^{d}$. Such results carry over to $K_{n}(d \nu ; x, y)$ by Theorem 3.3 . In one particular case, $\kappa=0$, the convergence holds for all $T^{d}$ as given in [12, Theorem 2.3]:

$$
\lim _{n \rightarrow \infty} \frac{1}{\left(\begin{array}{c}
n+d \\
n
\end{array}\right)} K_{n}\left(W_{0} ; x, x\right)=2^{d-k}, \quad x \in T_{k}^{d}, \quad 0 \leq k \leq d,
$$

where $T_{k}^{d}$ denotes the $k$-dimensional face of $T^{d}$, which contains elements of $T^{d}$ for which exactly $d-k$ inequalities in $T^{d}=\left\{x: x_{1} \geq 0, \ldots, x_{d+1} \geq 0\right\}$ becomes equalities. In particular, $T_{d}^{d}$ (when none of the inequalities become equality) is the interior of $T^{d}$, and 0 -dimensional face $T_{0}^{d}$ is the set of the vertices. Setting $\kappa=0$, so that $\varsigma=0$ and $\lambda=(d+1) / 2$, we see that

$$
P_{n}^{(\lambda-\varsigma-1 / 2, \varsigma-1 / 2)}\left(2 x_{i}-1\right)=P_{n}^{(d / 2,-1 / 2)}\left(2 x_{i}-1\right)=(-1)^{n} P_{n}^{(-1 / 2, d / 2)}\left(1-2 x_{i}\right),
$$

which is bounded by $c n^{-1 / 2}$ whenever $1-x_{i} \geq \varepsilon>0$. It follows then that

$$
\lim _{n \rightarrow \infty} \frac{1}{\left(\begin{array}{c}
n+d \\
n
\end{array}\right)} \sum_{i=1}^{d+1}\left[P_{n}^{(d / 2,-1 / 2)}\left(2 x_{i}-1\right)\right]^{2}= \begin{cases}0 & x \in T_{k}^{d}, k>0 \\
1 & x \in T_{0}^{d},\end{cases}
$$

upon using the fact that $P_{n}^{(a, b)}(1)=\left(\begin{array}{c}n+a \\ n\end{array}\right)$. By (3.10), we then end up with the following corollary.

Corollary 3.4. For $\kappa=0$,

$$
\lim _{n \rightarrow \infty} \frac{1}{\left(\begin{array}{c}
n+d \\
n
\end{array}\right)} K_{n}(d \nu ; x, x)= \begin{cases}2^{d-k}, & x \in T_{k}^{d}, k>0, \\
2^{d}+E_{d}, & x \in T_{0}^{d}\end{cases}
$$

where $E_{d}=c_{(d+1) / 2,0}=\Gamma(d / 2+1) \sqrt{\pi} /\left(\Gamma(d+1 / 2) 2^{d+1}\right)$.

Comparing to (3.11), the result shows the impact of the additional mass points at the vertices. More generally, if $\kappa_{i}=\varsigma>0$, then (3.10) shows that

$$
\begin{aligned}
K_{n}(d \nu ; x, x)-K_{n}\left(W_{\kappa} ; x, x\right)= & c_{\lambda, \varsigma}\left(1+\mathcal{O}\left(n^{-1}\right)\right) \\
& \times\left\{\begin{array}{cc}
\left(\begin{array}{c}
n+\lambda-\varsigma-1 / 2 \\
n
\end{array}\right), & x \in T_{0}^{d}, \\
2^{k}\left(\begin{array}{c}
n+\varsigma-1 / 2 \\
n
\end{array}\right), & x \in T_{k}^{d}, 1 \leq k \leq d-1,
\end{array}\right.
\end{aligned}
$$


since, for $x \in T^{d}, x_{i}=1$ only when $x=e_{i}$. In particular, we see that

$$
\lim _{n \rightarrow \infty} \frac{1}{\left(\begin{array}{c}
n+d \\
n
\end{array}\right)}\left[K_{n}(d \nu ; x, x)-K_{n}\left(W_{\kappa} ; x, x\right)\right]=0, \quad x \in T_{k}^{d}, \quad 1 \leq k \leq d,
$$

if $d>2 \varsigma-1$, whereas this limit is unbounded when $x \in T_{0}^{d}$.

\section{REFERENCES}

[1] M. Abramowitz, and I. A. Stegun, (Eds.). Handbook of Mathematical Functions with Formulas, Graphs, and Mathematical Tables, 9th printing. New York: Dover, 1972.

[2] C. F. Dunkl and Y. Xu, Orthogonal polynomials of several variables, Encyclopedia of Mathematics and its Applications 81. Cambridge University Press, 2001.

[3] L. Fernández, T. E. Pérez, M. A. Piñar, Y. Xu, Krall-type orthogonal polynomials in several variables, J. Comp. Appl. Math. (to appear).

[4] J. J. Guadalupe, M. Pérez, F. J. Ruiz, J. L. Varona, Asymptotic behaviour of orthogonal polynomials relative to measures with mass points. Mathematika 40 (1993), no. 2, 331-344.

[5] T. H. Koornwinder, Orthogonal polynomials with weight function $(1-x)^{\alpha}(1+x)^{\beta}+M \delta(x+$ 1) $+N \delta(x-1)$, Canad. Math. Bull. 27 (1984), no. 2, 205-214.

[6] M. A. Kowalski, The recursion formulas for orthogonal polynomials in $\mathrm{n}$ variables, SIAM J. Math. Anal. 13 (1982), 309-315.

[7] A. M. Krall, Orthogonal polynomials satisfying fourth order differential equations, Proc. Royal Soc. Edinburg Sect A 87 (1980/81), 271-288.

[8] G. Szegö, Orthogonal polynomials, 4th ed., American Mathematical Society Colloquium Publication 23, Providence RI, 1978.

[9] V. B. Uvarov, The connection between systems of polynomials that are orthogonal with respect to different distribution functions, USSR Computat. Math. and Math. Phys., 9 (1969), $25-36$.

[10] Y. Xu, On multivariable orthogonal polynomials, SIAM J. Math. Anal. 24 (1993) 783-794.

[11] Y. Xu, Summability of Fourier orthogonal series for Jacobi weight functions on the simplex in $R^{d}$. Proc. Amer. Math. Soc. 126 (1998), no. 10, 3027-3036.

[12] Y. Xu, Asymptotics of the Christoffel functions on a simplex in $\mathbb{R}^{d}$, J. Approx. Theory 99 (1999), no. 1, 122-133.

(A. M. Delgado) Departamento de Matemática Aplicada, Universidad de Granada, SPAIN

(L. Fernández) Departamento de Matemática Aplicada, and Instituto Carlos i de Física Teórica y Computacional, Universidad de Granada, Spain

(T. E. Pérez) Departamento de Matemática Aplicada, and Instituto Carlos I de Física Teórica y Computacional, Universidad de Granada, Spain

(M. A. Piñar) Departamento de Matemática Aplicada, and Instituto Carlos I de Física Teórica y Computacional, Universidad de Granada, Spain

(Y. Xu) Department of Mathematics, Universty of Oregon, USA 